\title{
Síntese e caracterização de nanopartículas de $\mathrm{Fe}_{3} \mathrm{O}_{4} @ \mathrm{SiO}_{2}$
}

\author{
Synthesis and characterization of the \\ $\mathrm{Fe}_{3} \mathrm{O}_{4} @ \mathrm{SiO}_{2}$ nanoparticles
}

Barbara Oliveira Tessarolli ${ }^{1}$, Paulo Vitor da Silva ${ }^{1}$ Emílio Carlos Gallardo ${ }^{1}$, Aroldo Geraldo Magdalena ${ }^{1}$

\footnotetext{
${ }^{1}$ Universidade Estadual Paulista - UNESP - Departamento de Química da Faculdade de Ciências, CP: 17033-360, Bauru, São Paulo, Brasil.

e-mail: aroldo.magdalena@unesp.br, emilio.gallardo@gmail.com, paulovictory@gmail.com, tessarollibarbara@gmail.com
}

\begin{abstract}
RESUMO
Neste trabalho foi estudada a síntese e caracterização das nanopartículas magnéticas de magnetita $\left(\mathrm{Fe}_{3} \mathrm{O}_{4}\right)$ através da co-precipitação e, sua posterior funcionalização pela sílica $\left(\mathrm{SiO}_{2}\right)$, realizada via metodologia solgel. A caracterização das nanopartículas foi feita por difratometria de raios X (DRX), microscopia eletrônica de transmissão (MET), espectroscopia de infravermelho com transformada de Fourier (FTIR) e medidas da variação de potencial zeta em função do $\mathrm{pH}$. Os resultados obtidos mostraram que a síntese das nanopartículas de $\mathrm{Fe}_{3} \mathrm{O}_{4}$ e $\mathrm{Fe}_{3} \mathrm{O}_{4} @ \mathrm{SiO}_{2}$ foi bem-sucedida, com uma alta homogeneidade de forma e tamanho, conforme resultados das análises das imagens obtidas por MET, inclusive com a formação de estruturas do tipo cascacaroço para as nanopartículas de $\mathrm{Fe}_{3} \mathrm{O}_{4} @ \mathrm{SiO}_{2}$. A funcionalização da superfície da magnetita foi comprovada a partir dos dados de FTIR e das medidas de potencial zeta. A funcionalização da superfície, pela sílica, ocasiona um aumento do tamanho das nanopartículas devido à introdução de uma casca inorgânica ao redor das nanopartículas de magnetita. As curvas de potencial zeta mostraram que com a adição de sílica, a estabilidade coloidal aumenta em relação à magnetita e este está relacionado à sílica funcionalizada que deve estar minimizando as energias superficiais ao redor das nanopartículas magnéticas.
\end{abstract}

Palavras-chave: magnetita, sílica, funcionalização, estrutura casca-caroço.

\section{ABSTRACT}

In this work the synthesis and characterization of the magnetic nanoparticles of magnetite $\left(\mathrm{Fe}_{3} \mathrm{O}_{4}\right)$ from the methodology via co-precipitation and its subsequent functionalization by silica $\left(\mathrm{SiO}_{2}\right)$, carried out via sol-gel methodology were studied. The characterization of the nanoparticles was performed by X-ray diffractometry (XRD), transmission electron microscopy (TEM), Fourier transform infrared spectroscopy (FTIR) and zeta potential variation as a function of $\mathrm{pH}$. The results showed that the synthesis of the $\mathrm{Fe}_{3} \mathrm{O}_{4}$ and $\mathrm{Fe}_{3} \mathrm{O}_{4} @ \mathrm{SiO}_{2}$ nanoparticles was successful, with a high homogeneity of shape and size, according to the results of the analysis of the images obtained by MET, including the formation of shell structures for the $\mathrm{Fe}_{3} \mathrm{O}_{4} @ \mathrm{SiO}_{2}$ nanoparticles. The functionalization of the magnetite surface was confirmed by the FTIR data and the zeta potential measurements. Surface functionalization by silica causes an increase in nanoparticle size due to the introduction of an inorganic shell around magnetite nanoparticles. The zeta potential curves showed that with the addition of silica, the colloidal stability increases in relation to the magnetite and this increase is related to the functionalized silica that should be minimizing the surface energies around the magnetic nanoparticles.

Keywords: Magnetite, silica, functionalization, core-shell structure

\section{INTRODUÇÃO}

As nanopartículas à base de óxido de ferro, como a magnetita $\left(\mathrm{Fe}_{3} \mathrm{O}_{4}\right)$ tem sido estudada por possuir uma propriedade magnética muito interessante, elas são superparamagnéticas e podem ser manipuladas, facilmente, pela aplicação de campos magnéticos [1], além disso, podem ser compostas de diferentes átomos ou íons com diferentes momentos magnéticos [1-2]. A utilização de nanopartículas de óxido de ferro em estudos in vitro ocorre há décadas e as nanopartículas mais pronunciadas são chamadas de nanopartículas de óxido de 
ferro com valência zero (nZVI), maghimita $\left(\gamma-\mathrm{Fe}_{2} \mathrm{O}_{3}\right)$ e magnetita $\left(\mathrm{Fe}_{3} \mathrm{O}_{4}\right)$, devido as suas biocompatibilidades já comprovadas [1,2].

Para as nanopartículas de $\mathrm{Fe}_{3} \mathrm{O}_{4}$ a adsorção física e química dos metais pesados já foi reportada. Entretanto, o mecanismo majoritário da remoção de contaminantes das nanopartículas de $\gamma-\mathrm{Fe}_{2} \mathrm{O}_{3}$ é pela adsorção física, enquanto que a adsorção química é observada para as nanopartículas de $\mathrm{Fe}_{3} \mathrm{O}_{4}$ [2].

$\mathrm{O}$ aumento do interesse das nanopartículas é particularmente devido à simplicidade de preparação e seu armazenamento estável na forma coloidal. Suspensões coloidais das nanopartículas magnéticas estabilizadas são chamadas de ferrofluidos e podem interagir com o campo magnético [1,3]. Estas também são de grande interesse, pois abordam uma vasta área do conhecimento incluindo fluídos magnéticos, catálise, biotecnologia/biomedicina, imagens de ressonância magnética, armazenamento de dados e remediação ambiental [4].

Entretanto, um problema inevitável associado com partículas neste intervalo de tamanho é a sua instabilidade ao longo do tempo. As partículas tendem a formar aglomerados para reduzir a energia associada com a área de superfície elevada para uma razão de volume das partículas nanométricas. Além disso, as nanopartículas descobertas são quimicamente ativas, facilmente oxidadas no ar, resultando geralmente em baixo magnetismo e dispersibilidade. Para muitas aplicações é crucial o desenvolvimento de estratégias de proteção para estabilizá-las quimicamente contra a degradação durante ou depois da síntese. Essas estratégias incluem o enxerto de revestimento ou com espécies orgânicas, incluindo surfactantes ou polímeros, ou revestimento com uma camada (filme) inorgânica, como a sílica ou carbono. É notável que em muitos casos de proteção a casca (shell) não apenas estabiliza as nanopartículas, mas pode também ser usada para futuras funcionalizações, por exemplo, com outras nanopartículas ou vários ligantes, dependendo da aplicação desejada [5].

$\mathrm{O}$ objetivo deste trabalho foi estudar a síntese e caracterização das nanopartículas de $\mathrm{Fe}_{3} \mathrm{O}_{4}$ recobertas com sílica e verificar as propriedades da adição da sílica, como recobrimento das nanopartículas.

\section{MATERIAIS E MÉTODOS}

Os reagentes utilizados para a síntese das nanopartículas foram: Cloreto de ferro (II) tetrahidratado (Merck), Cloreto de ferro (III) hexahidratado (Aldrich), hidróxido de amônio (Synth), etanol (Synth) e Tetraetilortosilicato (TEOS) (Aldrich).

A síntese das nanopartículas de $\mathrm{Fe}_{3} \mathrm{O}_{4} @ \mathrm{SiO}_{2}$ foi preparada em duas etapas: a primeira foi à síntese das nanopartículas magnéticas, usando-se uma mistura de $100 \mathrm{~mL}$ dos precursores, sendo 0,030 mols de solução de cloreto de ferro (III) hexahidratado e 0,015 mols de solução de cloreto de ferro II tetrahidratado. A esta mistura foi adicionada $10 \mathrm{~mL}$ de hidróxido de amônio 28\%, e deixada sob agitação constante durante 1 hora na temperatura ambiente com fluxo de nitrogênio. $\mathrm{O}$ precipitado final foi lavado diversas vezes com água destilada até obter $\mathrm{pH}$ igual a 7,0 [3,6]

Para a modificação da superfície das nanopartículas com a Sílica, realizado a partir do método de Stober com pequenas modificações [7,8], na qual as nanopartícular de $\mathrm{Fe}_{3} \mathrm{O}_{4}$ foram misturados em água destilada juntamente com tetraetilortosilicato (TEOS) em um ultrasonicador até obtenção de uma emulsão estável, Posteriormente, foi adicionado etanol e, em seguida, hidróxido de amônio. A mistura permaneceu em constante agitação durante 4 horas, em temperatura ambiente. As nanopartículas de $\mathrm{Fe}_{3} \mathrm{O}_{4} @ \mathrm{SiO}_{2}$ foi separadas com auxílio de um imã e lavadas diversas vezes com água destilada e, em seguida, acetona. O precipitado foi seco em estufa com circulação forçada de ar na temperatura de $60^{\circ} \mathrm{C}$.

As nanopartículas foram caracterizadas usando um difratômetro de raios-X Rigaku-Rint 2000 com radiação $\mathrm{CuK}_{\alpha}$. As imagens por microscopia eletrônica de transmissão (TEM) foram obtidas com um Microscópio Philips CM-200. Os espectros de infravermelho com transformada de Fourrier foram medidos em um espectrômetro Vertex 70 (Bruker Instruments). As medidas de potencial zeta foram realizadas com o equipamento Zetasizer Nano ZS system (Malvern Instruments).

\section{RESULTADOS E DISCUSSÃO}

A Figura 1-a mostra os difratogramas de raios-X para as nanopartículas de $\mathrm{Fe}_{3} \mathrm{O}_{4}$ e $\mathrm{Fe}_{3} \mathrm{O}_{4} @ \mathrm{SiO}_{2}$. Neles podese verificar os planos cristalográficos referentes à difração da magnetita, tanto para as nanopartículas de $\mathrm{Fe}_{3} \mathrm{O}_{4}$ quanto para as de $\mathrm{Fe}_{3} \mathrm{O}_{4} @ \mathrm{SiO}_{2}$. A partir destes difratogramas foi possível verificar que as nanopartículas obtidas não sofreram oxidação, pois não foram observados planos cristalográficos referentes à maghmita $\left(\gamma-\mathrm{Fe}_{2} \mathrm{O}_{3}\right)$. Segundo Gupta e colaboradores [1], para inibir a oxidação das nanopartículas, geralmente usam-se 
moléculas inorgânicas ou orgânicas durante o processo da precipitação. Neste caso, a sílica ajudou na proteção do processo de oxidação. O controle cinético da oxidação das espécies ferrosas pode ser diminuído com a utilização de sistema para retirar o oxigênio do meio reacional, isso pode ser obtido, borbulhando gás $\mathrm{N}_{2}$ durante a síntese. A relação molar ideal para a síntese da magnetita é $2: 1$ em relação a $\mathrm{Fe}^{3+}: \mathrm{Fe}^{2+}$ e para garantir esta relação é utilizada atmosfera inerte no meio reacional e este controle é muito importante, pois pode modificar as propriedades de superfície destes materiais, uma vez que a propriedade magnética tende a diminuir com a oxidação.
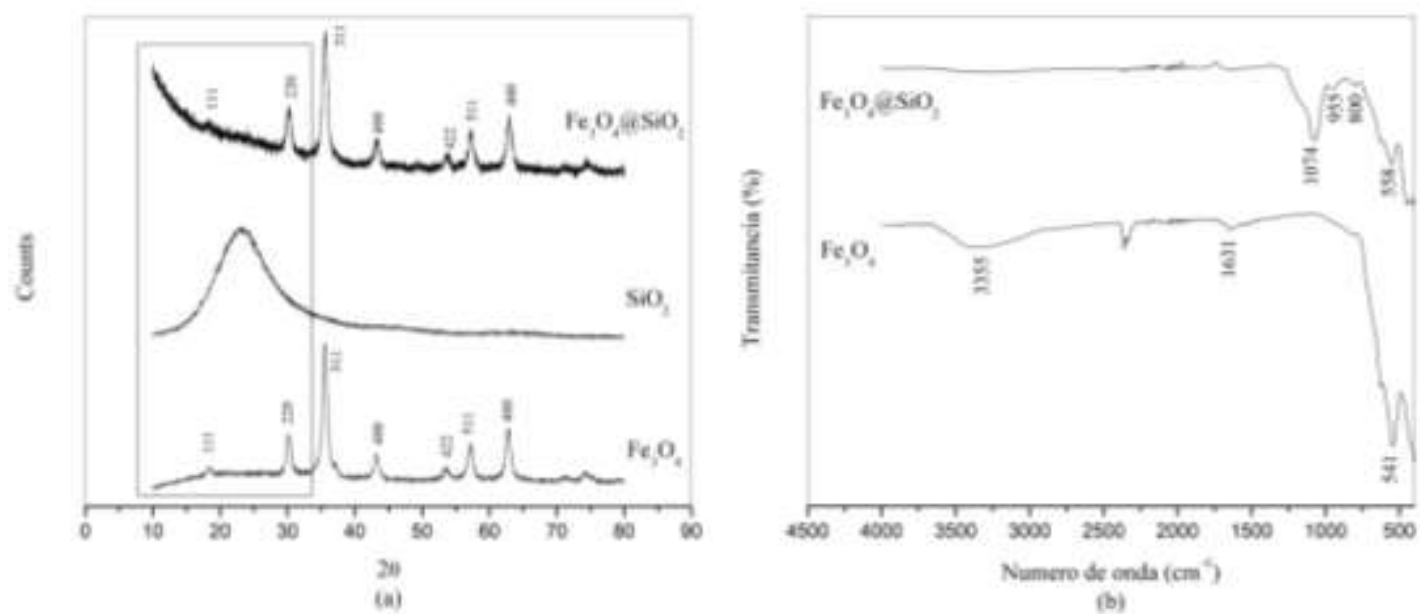

Figura 1: (a) Difratogramas de raios-X obtidos para as nanoparticulas $\mathrm{Fe}_{3} \mathrm{O}_{4}, \mathrm{SiO}_{2}$ e $\mathrm{Fe}_{3} \mathrm{O}_{4} @ \mathrm{SiO}_{2}$. Os padrões de difração, segundo a base de dados JCPDS para a magnetita é (JCPDS card no. 89-0688) e para a maghmita é (JCPDS card no. 89-0597). (b) Espectros de infravermelho obtidos para a nanopartícula de $\mathrm{Fe}_{3} \mathrm{O}_{4} \mathrm{e} \mathrm{Fe}_{3} \mathrm{O}_{4} @ \mathrm{SiO}_{2}$.

Na figura 1-a, também é possível observar que no intervalo de $2 \theta=10-20^{\circ}$ apresenta uma declive da linha base. Este evento deve estar relacionado com a camada de sílica amorfa ao redor das nanopartículas de magnetita, o mesmo comportamento foi verificado pelo trabalho de Chae e colaboradores [8].

Para confirmar este resultado do desvio da linha base, foram realizadas análises de espectroscopia no infravermelho, mostrados na Figura 1-b e a Tabela 1 mostra a relação das bandas obtidas. Nestes interferogramas é possível observar o surgimento de novas bandas, em comparação com as nanopartículas de $\mathrm{Fe}_{3} \mathrm{O}_{4}$. Estas estão relacionadas com as vibrações da ligação O-Si-O [7,8] $\left(1077,959\right.$ e $\left.812 \mathrm{~cm}^{-1}\right)$. Esse resultado confirma a funcionalização da superfície das nanopartículas com o grupo sílica. Outro indicativo mostrado nos dados de infravermelho está relacionado com o deslocamento de $17 \mathrm{~cm}^{-1}$ da banda relacionada à ligação Fe-O, que deve estar relacionada com a interação da sílica Fe-O-Si-O [9]. Os grupos $-\mathrm{OH}$ da superfície da magnetita foram utilizados para a ligação química ente a magnetita e a sílica.

Tabela 1: Descrição das bandas de FTIR do espectro mostrado na figura 1-b.

\begin{tabular}{|c|c|}
\hline $\mathrm{Fe}_{3} \mathrm{O}_{4}$ & $\mathrm{Fe}_{3} \mathrm{O}_{4} @ \mathrm{SiO}_{2}$ \\
\hline$\left(541 \mathrm{~cm}^{-1}\right) \mathrm{Fe}-\mathrm{O}$ & $\left(558 \mathrm{~cm}^{-1}\right) \mathrm{Fe}-\mathrm{O}$ \\
\hline- & $\left(800 / 955 / 1074 \mathrm{~cm}^{-1}\right) \mathrm{O}-\mathrm{Si}-\mathrm{O}$ \\
\hline$\left(1631 \mathrm{~cm}^{-1}\right) v-\left(\mathrm{H}_{2} \mathrm{O}\right)$ & - \\
\hline$\left(3355 \mathrm{~cm}^{-1}\right) v-(\mathrm{O}-\mathrm{H})$ & - \\
\hline
\end{tabular}

As micrografias eletrônicas de transmissão (TEM) mostradas nas Figuras 2 e 3 mostram que as nanoestruturas apresentam uma alta homogeneidade de forma e tamanhos. As TEM (Fig. 2-a e 2-b) apresentam tamanho médio das nanopartículas em torno $12 \pm 3 \mathrm{~nm}$ e $20 \pm 5 \mathrm{~nm}$, como verificado pelos histogramas da figura $3^{9}$.

Nestas imagens de MET apresentadas na figura 2 (b-c) fica claro o recobrimento da sílica ao redor das nanopartículas magnéticas e a figura 2-c mostra uma ampliação com maiores detalhes da estrutura coreshell (casca-caroço), onde as nanopartículas magnéticas são o core, e a sílica forma uma casca protetora do core metálico. Nesta figura, a estrutura core-shell apresenta tamanho em torno de $28 \mathrm{~nm}$. O core magnético apresenta tamanho médio em torno de $14 \mathrm{~nm}$, enquanto que o recobrimento apresenta em torno de $7 \mathrm{~nm}$ de espessura ao redor das nanopartículas magnéticas. 

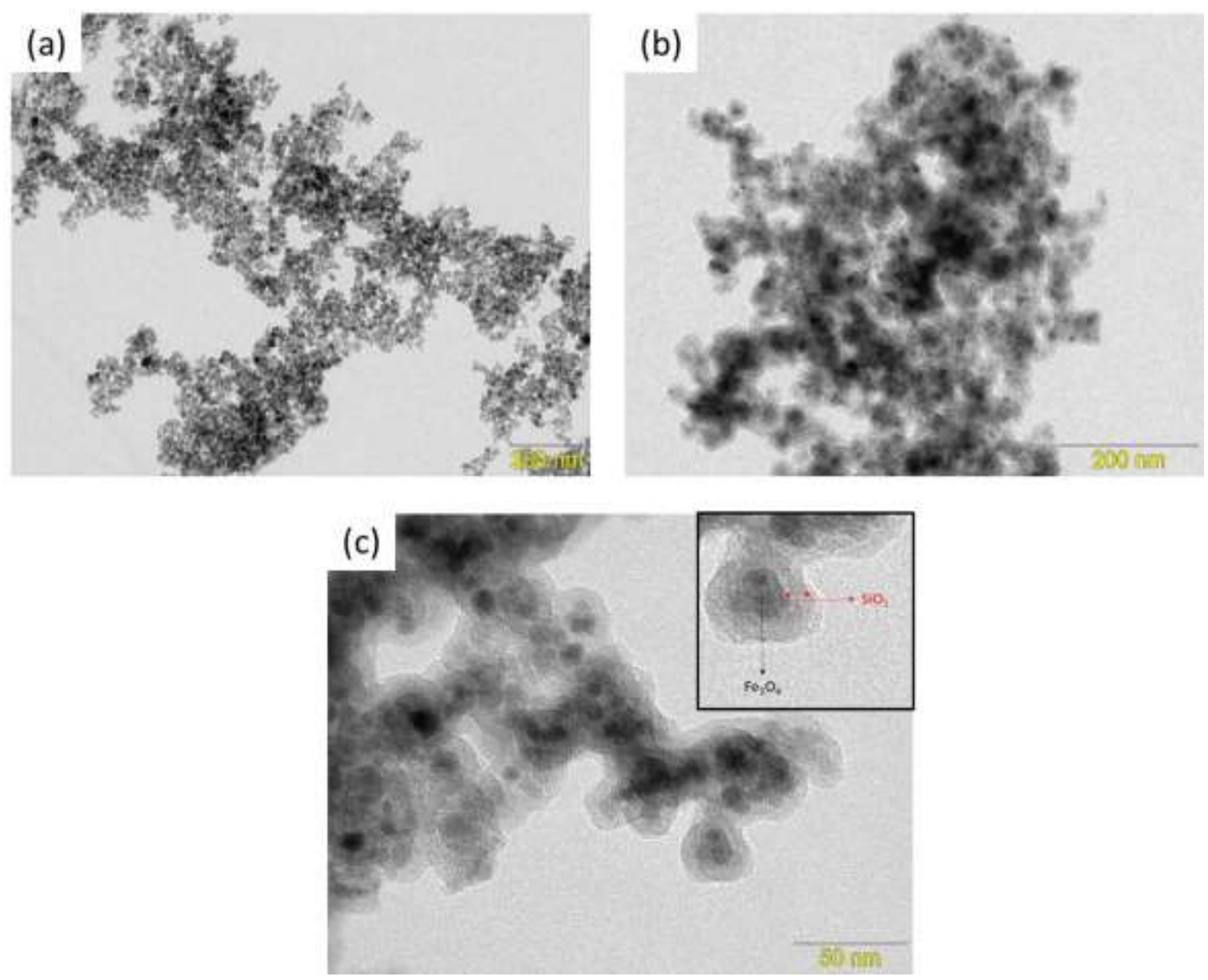

Figura 2: Microscopia eletrônica de transmissão obtida para (a) nanopartículas de $\mathrm{Fe}_{3} \mathrm{O}_{4}$ e (b) nanopartículas de $\mathrm{Fe}_{3} \mathrm{O}_{4} @ \mathrm{SiO}_{2}$. (c) Microscopia eletrônica de transmissão para a nanopartícula de $\mathrm{Fe}_{3} \mathrm{O}_{4} @ \mathrm{SiO}_{2}$ com uma maior magnificação, e em destaque a ampliação de uma estrutura do tipo core-shell.

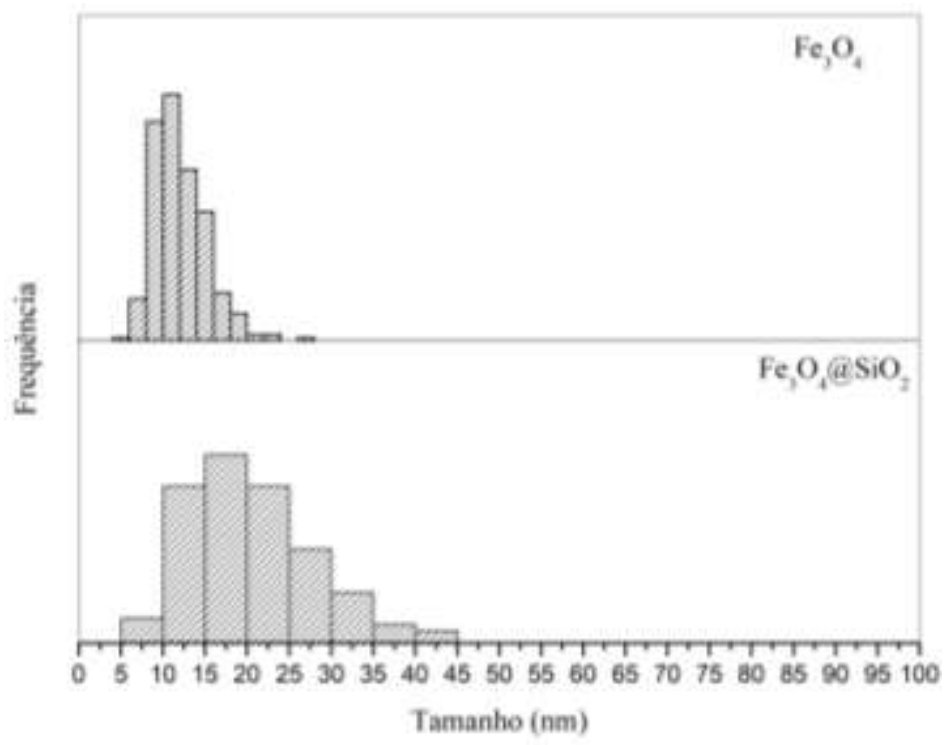

Figura 3: Histogramas das nanopartículas de $\mathrm{Fe}_{3} \mathrm{O}_{4}[6]$ e $\mathrm{Fe}_{3} \mathrm{O}_{4} @ \mathrm{SiO}_{2}$ obtidos a partir das imagens da figura 2.

A figura 4 mostra as curvas de potencial zeta $(\zeta)$ em função do $\mathrm{pH}$ para as nanopartículas de $\mathrm{Fe}_{3} \mathrm{O}_{4} \mathrm{e}$ $\mathrm{Fe}_{3} \mathrm{O}_{4} @ \mathrm{SiO}_{2}$. A adição do grupo sílica modifica o ponto isoelétrico da magnetita de 5,7 para em torno de 2,0 $\mathrm{mV}$. Além disso, pode-se verificar que o potencial zeta da nanopartículas $\mathrm{Fe}_{3} \mathrm{O}_{4} @ \mathrm{SiO}_{2}$ encontra-se em valo- 
res negativos e desta forma aumenta a estabilidade coloidal destas nanopartículas do intervalo de $\mathrm{pH}$ de 4 a 11, enquanto as nanopartículas de $\mathrm{Fe}_{3} \mathrm{O}_{4}$ apresentam pequena estabilidade de $\mathrm{pH}$ nos intervalos de 2,0 a 3,5 e entre 9 a 11 unidades de $\mathrm{pH}$. Estes resultados sugerem que a variação do ponto isoelétrico é ocasionada pela distribuição de cargas na superfície das nanopartículas e que a densidade de carga relacionada à sílica é predominante e está de acordo com a discussão proposta pela espectroscopia no IR. Este aumento de estabilidade deve estar relacionado com a casca inorgânica de $\mathrm{SiO}_{2}$ que está protegendo as nanopartículas de $\mathrm{Fe}_{3} \mathrm{O}_{4}$. Estes resultados são importantes, pois a funcionalização melhorou a estabilidade coloidal acima do $\mathrm{pH}$ igual a 4. As cargas de superfície abaixo do ponto isoelétrico são basicamente positivas e acima negativas. Desta forma, a introdução da sílica às nanopartículas de magnetita apresentam cargas superficiais negativas em todo o intervalo de pH estudado. Além disso, a casca inorgânica auxilia na melhoria da estabilidade coloidal minimizando a energia superficial das nanopartículas magnéticas. A introdução do grupo sílica torna a magnetita multifuncional, podendo atuar na adsorção de corantes e íons metálicos, além disso, a adição da sílica pode alterar o band gap da magnetita e sendo assim melhorar as propriedades ópticas do material $[7,8]$ podendo atuar como um fotodegradador [9] de corantes e compostos orgânicos como os fármacos. Além de todas as propriedades supracitadas, a vantagem do uso das nanopartículas magnéticas está relacionada à sua fácil remoção dos meios reacionais com o auxílio de um campo magnético externo.

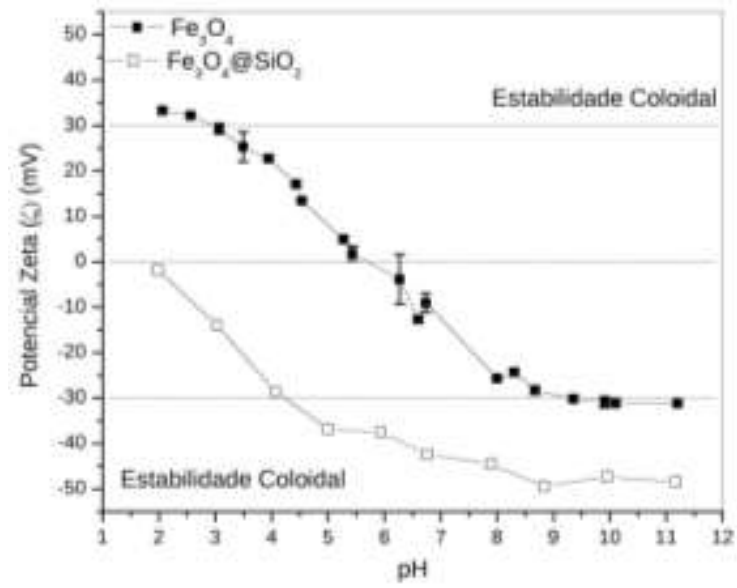

Figura 4: Variação do potencial zeta em função do $\mathrm{pH}$ para as nanoparticulas de $\mathrm{Fe}_{3} \mathrm{O}_{4} \mathrm{e} \mathrm{Fe}_{3} \mathrm{O}_{4} @ \mathrm{SiO}_{2}$.

\section{CONCLUSÕES}

Os resultados obtidos mostraram que a síntese das nanopartículas de $\mathrm{Fe}_{3} \mathrm{O}_{4}$ e $\mathrm{Fe}_{3} \mathrm{O}_{4} @ \mathrm{SiO}_{2}$ foi bem sucedida, obtendo estruturas com uma alta homogeneidade de tamanho e forma, conforme resultados das análises das imagens obtidas por MET. A funcionalização da superfície da magnetita foi comprovada pelo espectro de FTIR e curva de potencial zeta, onde foi possível observar um aumento do tamanho devido à introdução de uma casca inorgânica ao redor das nanopartículas de magnetita. As curvas de potencial zeta mostraram que, com a adição de sílica, a estabilidade coloidal aumenta em relação à magnetita e este está relacionado à funcionalização promovida pela sílica, onde esta deve estar minimizando as energias superficiais ao redor das nanopartículas magnéticas.

\section{BIBLIOGRAFIA}

[1] GUPTA, A. K., GUPTA, M. "Synthesis and surface engineering of iron oxide nanoparticles for biomedical applications” Biomaterials, v. 26, pp. 3995-4021, 2005.

[2] TANG, S. C. N., LO, I. M. C. "Magnetic nanoparticles: Essential factors for sustainable environmental applications" Water Research, v. 47, pp. 2613-2632, 2013.

[3] BINI, R. A., MARQUES, R. F. C., et al. "Synthesis and functionalization of magnetite nanoparticles with different amino-functional alkoxysilanes", Journal of Magnetism and Magnetic Materials, v. 324, pp. 534$539,2012$.

[4] LU, A., SALABAS, E. L., et al. "Magnetic nanoparticles: Synthesis, protection, functionalization, and application”, Angewandte Chemie, v. 46, pp. 1222-1244, 2007. 
[5] ZHANG, J., ZHAI, S., et al., "Pb(II) removal of $\mathrm{Fe}_{3} \mathrm{O}_{4} @ \mathrm{SiO}_{2}-\mathrm{NH}_{2}$ core-shell nanomaterials prepared via a controllable sol-gel process", Chemical Engineering Journal, v. 215-216, pp. 461-471, 2013.

[6] MAGDALENA, A. G., SILVA, I. M. B., et al., "EDTA-functionalized Fe3O4 nanoparticles" Journal of Physics and Chemistry of Solids, v. 113, pp. 5-10, 2018.

[7] LU, P., ZHANG, J. L., et al. "Synthesis and characteristic of the $\mathrm{Fe}_{3} \mathrm{O}_{4} @ \mathrm{SiO}_{2} @ \mathrm{Eu}(\mathrm{DBM})_{3} \cdot 2 \mathrm{H}_{2} \mathrm{O} / \mathrm{SiO}_{2}$ luminomagnetic microsphere with core-shell structure", Talanta, v.82, pp. 450-457, 2010.

[8] CHAE, H. S., KIM, S. D., et al. "Core-shell structured $\mathrm{Fe}_{3} \mathrm{O}_{4} @ \mathrm{SiO}_{2}$ nanoparticles fabricated by sol-gel method and their magnetorheology" Colloid and Polymer Science, v. 294, n. 4, pp. 647-655, 2016.

[9] TEXEIRA, S., MORA, H., et al., "Photocatalytic degradation of recalcitrante micropollutants by resusable $\mathrm{Fe}_{3} \mathrm{O}_{4} / \mathrm{SiO}_{2} / \mathrm{TiO}_{2}$ particles" Journal of Photochemistry and Photobiology A: Chemistry, v. 345, pp. 27-35, 2017.

\section{ORCID}

Barbara Oliveira Tessarolli

http://orcid.org/0000-0002-4036-6520

Emílio Carlos Galhardo

https://orcid.org/0000-0002-8067-5301

Paulo Vitor da Silva

https://orcid.org/0000-0002-2127-8226

Aroldo Geraldo Magdalena

http://orcid.org/0000-0003-3385-2106 